accepted in clinical practice is practically excluded. At the same time, it is necessary to take into account the anatomical and physiological characteristics of the child's body and the psycho-emotional state of the children.

To assess the severity of the child's condition prior to the development of classic clinical signs, damage qualimetry is recommended, which is essentially a quantitative characteristic of the injury shoginess. The scoring of polytrauma is defined as the sum of points of private injury. The real threat of traumatic shock arises when the severity of damage exceeds 6 points.

The standard for providing therapeutic measures in children with traumatic shock includes: Pain relief; Venous access; Infusion therapy; Transport immobilization; Medical correction; Oxygen therapy.

The system of rendering psychological and psychiatric assistance to children and adolescents allows rendering differentiated psychiatric and psychological assistance, as well as carrying out rehabilitation activities in a more remote period. The training of pediatricians in the field of disaster psychiatry should be considered the most important condition for the further improvement of the entire system of assistance to victims and those affected by emergencies.

\section{P284 A CASE OF FAMILIAL CRANIAL DIABETES INSIPIDUS - IT'S ALL IN THE HISTORY}

${ }^{1}$ Tracey A Conlon*, ${ }^{1}$ Sinead Moloney, ${ }^{2}$ Colm Costigan, ${ }^{1}$ Nuala P Murphy. ${ }^{1}$ Department of Endocrinology, Children's University Hospital, Temple Street., Dublin, Ireland; ${ }^{2}$ Department of Endocrinology, Our Lady's Children's Hospital, Crumlin., Dublin, Ireland

\subsection{6/archdischild-2019-epa.634}

Aims Cranial diabetes insipidus is characterised by polyuria and polydipsia secondary to partial or complete deficiency of antidiuretic hormone. Although in most patients non-hereditary causes underlie the disorder, rarer genetic defects in arginine vasopressin (AVP) synthesis have been identified. We describe the case of a 5 year old girl who posed a significant diagnostic challenge, with a suggestive history and strong family history of cranial diabetes insipidus, but non diagnostic biochemistry.

Methods Our patient was reassessed and AVP gene testing was performed.

Results A 5 year old girl, presented with a four year history of polyuria, polydipsia and associated enuresis. Family history was remarkable for suspected familial cranial diabetes insipidus responsive to desmopressin, in the patient's mother, maternal uncle and maternal grandfather. Genetic testing had not previously been undertaken. Our patient had previously been investigated with water deprivation test at age 3 years and was found to have normal biochemistry, without polyuria during the test. She represented at the age of 5 years to our service with ongoing symptoms and underwent repeat water deprivation testing. Serum sodium and osmolality remained normal (max $140 \mathrm{mmol} / \mathrm{l}$ and $285 \mathrm{mmol} / \mathrm{kg}$ respectively) with water deprivation but she did not concentrate her urine (urine osmolality $222 \mathrm{mmol} / \mathrm{kg}$ at the end of water deprivation test). AVP gene testing was requested and identified a heterozygous pathogenic missense mutation c.61T $>\mathrm{C}$, confirming a diagnosis of familial cranial diabetes insipidus. The same mutation was identified in the patient's mother, suggesting autosomal dominant inheritance. She was commenced on desmopressin with excellent response.
Conclusions Genetic testing may be a useful aid in the diagnosis of inherited cranial diabetes insipidus. Since these patients have progressive loss of AVP, they may initially respond normally to water deprivation testing. If the index of suspicion remains high, genetic testing is recommended to guide treatment.

\section{P285 THE ASSOCIATION OF CONGENITAL ANOMALIES IN PATIENTS WITH CONGENITAL HYPOTHYROIDISM IN GOVERNMENT TERTIARY CARE CENTERS UNDER DUBAI HEALTH AUTHORITY, UAE: 2000-2015, A RETROSPECTIVE CROSS - SECTIONAL STUDY}

Fatima Ali Mazahir*, Manal Mustafa Khadora. Latifa Hospital Dubai Health Authority, Dubai, UAE

\subsection{6/archdischild-2019-epa.635}

Background Congenital hypothyroidism $(\mathrm{CH})$ is the most common endocrine abnormality of the new- born with associated extra- thyroidal anomalies (ETAs) been documented in the literature at varying rates. Majority of the patients are diagnosed with $\mathrm{CH}$ at an earlier age due to the implementation of the neonatal screening program in many countries of the world. Congenital heart diseases (CHD) are the most frequently reported extra thyroidal anomaly. No data on such associations have been reported from the Middle Eastern region.

Aim To demonstrate the number and describe the variety of congenital extra- thyroidal anomalies associated with congenital hypothyroidism in babies born in UAE.

Methodology The study was designed as a retrospective, crosssectional study in patients with confirmed diagnosis of congenital hypothyroidism. 204 patients with confirmed congenital hypothyroidism from two tertiary government centres in Dubai under Dubai Healthy Authority with specialized paediatric endocrinology units were included in the study. Patients with Down syndrome, preterms $<35$ weeks gestation and babies with TORCH infections were subsequently excluded from the study.

Results 39\% of the included subjects with congenital hypothyroidism had associated extra- thyroidal anomalies. Out of these $25 \%$ had single ETA, 12\% had multiple ETAs and 2\% hadassociated syndromes. The total sample had a male to female ratio of 1.1:1 and a significant proportion of males from the Arab population of UAE and Middle- east were affected with $\mathrm{CH}$ as compared to other ethnic groups ( $\mathrm{p}=$ 0.03). The most common aetiological cause of $\mathrm{CH}$ was determined to be dyshormonogenesis (55\%) followed by dysgenesis (45\%). Amongst the cohort of dysgenesis, males with ectopic lingual thyroid gland were affected more with ETAs as compared to females of the same cohort $(\mathrm{p}=0.02)$. The most common ETAs were congenital heart disease (16\%) followed by urogenital tract anomalies (14\%). A wide spectrum of multiple ETAs was observed as well as a number of syndromes including Sanjad Sakati syndrome, Turner syndrome, William syndrome, VATER association and Congenital Disorder of Glycosylation.

Conclusion The study detected a higher rate of ETAs associated with congenital hypothyroidism in the included population subset with noted differences in the gender, ethnic distribution and etiological types of $\mathrm{CH}$ as compared to what 